\title{
On relationships between $q$-product identities and combinatorial partition identities
}

\author{
M.P. Chaudhary, Sangeeta Chaudhary, Sonajharia Minz
}

\begin{abstract}
Andrews et al. [2] discussed about the combinatorial partition identities. We aim to present some relationships between $q$-product identities and combinatorial partition identities, by using and combining known formulas.
\end{abstract}

\section{Introduction And Preliminaries}

Throughout this paper, $\mathbb{N}, \mathbb{Z}$, and $\mathbb{C}$ denote the sets of positive integers, integers, and complex numbers, respectively, and $\mathbb{N}_{0}:=\mathbb{N} \cup\{0\}$. The following $q$-notations are recalled (see, e.g., [15, Chapter 6]): The $q$-shifted factorial $(a ; q)_{n}$ is defined by

$$
(a ; q)_{n}:=\left\{\begin{array}{cc}
1, & n=0, \\
\prod_{k=0}^{n-1}\left(1-a q^{k}\right), & n \in \mathbb{N},
\end{array}\right.
$$

where $a, q \in \mathbb{C}$ and it is assumed that $a \neq q^{-m}\left(m \in \mathbb{N}_{0}\right)$. We also write

$$
(a ; q)_{\infty}:=\prod_{k=0}^{\infty}\left(1-a q^{k}\right)=\prod_{k=1}^{\infty}\left(1-a q^{k-1}\right), \quad(a, q \in \mathbb{C} ;|q|<1) .
$$

It is noted that, when $a \neq 0$ and $|q| \geq$, the infinite product in (1) diverges. So, whenever $(a ; q)_{\infty}$ is involved in a given formula, the constraint $|q|<1$ will be tacitly assumed.

The following notations are also frequently used:

$$
\left(a_{1}, a_{2}, \ldots, a_{m} ; q\right)_{n}:=\left(a_{1} ; q\right)_{n}\left(a_{2} ; q\right)_{n} \cdots\left(a_{m} ; q\right)_{n}
$$

2010 Mathematics Subject Classification. Primary 05A30, 11F27; Secondary 05A17, $11 \mathrm{P} 83$.

Key words and phrases. Combinatorial partition identities, Jacobi's triple-product identity, Ramanujan's theta functions, q-Product identities, Combinatorial partition identities, Euler's Pentagonal Number Theorem, Rogers-Ramanujan continued fraction, Rogers-Ramanujan identities.

Full paper. Received 31 January 2020, revised 24 February 2020, accepted 9 March 2020, available online 4 May 2020. 
and

$$
\left(a_{1}, a_{2}, \ldots, a_{m} ; q\right)_{\infty}:=\left(a_{1} ; q\right)_{\infty}\left(a_{2} ; q\right)_{\infty} \cdots\left(a_{m} ; q\right)_{\infty}
$$

Ramanujan defined the general theta function $f(a, b)$ as follows (see, for details, [3, p. 31, Eq. (18.1)] and [4], see also [13]):

$$
\begin{aligned}
f(a, b) & =1+\sum_{n=1}^{\infty}(a b)^{\frac{n(n-1)}{2}}\left(a^{n}+b^{n}\right) \\
& =\sum_{n=-\infty}^{\infty} a^{\frac{n(n+1)}{2}} b^{\frac{n(n-1)}{2}}=f(b, a), \quad(|a b|<1) .
\end{aligned}
$$

We find from (2) that

$$
f(a, b)=a^{\frac{n(n+1)}{2}} b^{\frac{n(n-1)}{2}} f\left(a(a b)^{n}, \quad b(a b)^{-n}\right)=f(b, a), \quad(n \in \mathbb{Z}) .
$$

Ramanujan also rediscovered the Jacobi's famous triple-product identity (see [3, p. 35, Entry 19]):

$$
f(a, b)=(-a ; a b)_{\infty}(-b ; a b)_{\infty}(a b ; a b)_{\infty},
$$

which was first proved by Gauss.

Several $q$-series identities emerging from Jacobi's triple-product identity (3) are worthy of note here (see [3, pp. 36-37, Entry 22]):

$$
\begin{aligned}
\phi(q) & :=\sum_{n=-\infty}^{\infty} q^{n^{2}}=1+2 \sum_{n=1}^{\infty} q^{n^{2}}= \\
& =\left\{\left(-q ; q^{2}\right)_{\infty}\right\}^{2}\left(q^{2} ; q^{2}\right)_{\infty}=\frac{\left(-q ; q^{2}\right)_{\infty}\left(q^{2} ; q^{2}\right)_{\infty}}{\left(q ; q^{2}\right)_{\infty}\left(-q^{2} ; q^{2}\right)_{\infty}} \\
& \psi(q):=f\left(q, q^{3}\right)=\sum_{n=0}^{\infty} q^{\frac{n(n+1)}{2}}=\frac{\left(q^{2} ; q^{2}\right)_{\infty}}{\left(q ; q^{2}\right)_{\infty}}, \\
f(-q) & :=f\left(-q,-q^{2}\right)=\sum_{n=-\infty}^{\infty}(-1)^{n} q^{\frac{n(3 n-1)}{2}}= \\
& =\sum_{n=0}^{\infty}(-1)^{n} q^{\frac{n(3 n-1)}{2}}+\sum_{n=1}^{\infty}(-1)^{n} q^{\frac{n(3 n+1)}{2}}=(q ; q)_{\infty} .
\end{aligned}
$$

Equation (6) is known as Euler's Pentagonal Number Theorem. The following $q$-series identity:

$$
(-q ; q)_{\infty}=\frac{1}{\left(q ; q^{2}\right)_{\infty}}=\frac{1}{\chi(-q)}
$$

provides the analytic equivalence of Euler's famous theorem: The number of partitions of a positive integer $n$ into distinct parts is equal to the number of partitions of $n$ into odd parts. 
We also recall the Rogers-Ramanujan continued fraction of $R(q)$ :

$$
\begin{aligned}
R(q) & :=q^{\frac{1}{5}} \frac{H(q)}{G(q)}=q^{\frac{1}{5}} \frac{f\left(-q,-q^{4}\right)}{f\left(-q^{2},-q^{3}\right)}=q^{\frac{1}{5}} \frac{\left(q ; q^{5}\right)_{\infty}\left(q^{4} ; q^{5}\right)_{\infty}}{\left(q^{2} ; q^{5}\right)_{\infty}\left(q^{3} ; q^{5}\right)_{\infty}}= \\
& =\frac{q^{\frac{1}{5}}}{1+\frac{q}{1+\frac{q^{2}}{1+\frac{q^{3}}{1+\cdots}}}}, \quad(|q|<1) .
\end{aligned}
$$

Here $G(q)$ and $H(q)$ are widely investigated Roger-Ramanujan identities defined by

$$
\begin{aligned}
G(q) & :=\sum_{n=0}^{\infty} \frac{q^{n^{2}}}{(q ; q)_{n}}=\frac{f\left(-q^{5}\right)}{f\left(-q,-q^{4}\right)}= \\
& =\frac{1}{\left(q ; q^{5}\right)_{\infty}\left(q 4 ; q^{5}\right)_{\infty}}=\frac{\left(q^{2} ; q^{5}\right)_{\infty}\left(q^{3} ; q^{5}\right)_{\infty}\left(q^{5} ; q^{5}\right)_{\infty}}{(q ; q)_{\infty}} ; \\
H(q) & :=\sum_{n=0}^{\infty} \frac{q^{n(n+1)}}{(q ; q)_{n}}=\frac{f\left(-q^{5}\right)}{f\left(-q^{2},-q^{3}\right)}= \\
& =\frac{1}{\left(q^{2} ; q^{5}\right)_{\infty}\left(q^{3} ; q^{5}\right)_{\infty}}=\frac{\left(q ; q^{5}\right)_{\infty}\left(q^{4} ; q^{5}\right)_{\infty}\left(q^{5} ; q^{5}\right)_{\infty}}{(q ; q)_{\infty}}
\end{aligned}
$$

and the functions $f(a, b)$ and $f(-q)$ are given in (2) and (6), respectively. For a detailed historical account of (and for various investigated developments stemming from) the Rogers-Ramanujan continued fraction (7) and identities (8) and (9), the interested reader may refer to the monumental work [3, p. 77 et seq.] (see also [13, 15]).

The following continued fraction was recalled in [14, p. 5, Eq. (2.8)] from an earlier work cited therein: For $|q|<1$,

$$
=\frac{\left(q^{2} ; q^{2}\right)_{\infty}(-q ; q)_{\infty}=\frac{\left(q^{2} ; q^{2}\right)_{\infty}}{\left(q ; q^{2}\right)_{\infty}}=}{1-\frac{1}{1+\frac{q(1-q)}{1-\frac{q^{3}}{1+\frac{q^{2}\left(1-q^{2}\right)}{1-\frac{q^{5}}{1+\frac{q^{3}\left(1-q^{3}\right)}{1-\cdots}}}}}},}
$$




$$
\begin{gathered}
\frac{\left(q ; q^{5}\right)_{\infty}\left(q^{4} ; q^{5}\right)_{\infty}}{\left(q^{2} ; q^{5}\right)_{\infty}\left(q^{3} ; q^{5}\right)_{\infty}}=\frac{1}{1+\frac{q}{1+\frac{q^{2}}{1+\frac{q^{3}}{1+\frac{q^{4}}{1+\frac{q^{5}}{1+\cdots}}}}}} \\
C(q):=\frac{\left(q^{2} ; q^{5}\right)_{\infty}\left(q^{3} ; q^{5}\right)_{\infty}}{\left(q ; q^{5}\right)_{\infty}\left(q^{4} ; q^{5}\right)_{\infty}}=1+\frac{q}{1+\frac{q^{2}}{1+\frac{q^{3}}{1+\frac{q^{4}}{1+\frac{q^{5}}{1+\cdots}}}}} .
\end{gathered}
$$

Andrews et al. [2] investigated new double summation hypergeometric $q$ series representations for several families of partitions and further explored the role of double series in combinatorial partition identities by introducing the following general family:

$$
R(s, t, l, u, v, w):=\sum_{n=0}^{\infty} q^{s\left(\begin{array}{l}
n \\
2
\end{array}\right)+t n} r(l, u, v, w ; n),
$$

where

$$
r(l, u, v, w: n):=\sum_{j=0}^{\left[\frac{n}{u}\right]}(-1)^{j} \frac{q^{u v\left(\begin{array}{c}
j \\
2
\end{array}\right)+(w-u l) j}}{(q ; q)_{n-u j}\left(q^{u v} ; q^{u v}\right)_{j}} .
$$

The following interesting special cases of (10) are recalled (see [2, p. 106, Theorem 3], see also [13]):

$$
\begin{gathered}
R(2,1,1,1,2,2)=\left(-q ; q^{2}\right)_{\infty}, \\
R(2,2,1,1,2,2)=\left(-q^{2} ; q^{2}\right)_{\infty}, \\
R(m, m, 1,1,1,2)=\frac{\left(q^{2 m} ; q^{2 m}\right)_{\infty}}{\left(q^{m} ; q^{2 m}\right)_{\infty}} .
\end{gathered}
$$

Here, we aim to present certain interrelations between $q$-product identities and combinatorial partition identities associated with the identities (4)-(6) and (11)-(13). 


\section{Sets of Preliminary Results}

Here we recall the following results for the verification of the main results in section 3 (see [1, Theorem 5.1; 3, Entry 51, p. 204; 12, Theorem 3.1]).

$$
\begin{gathered}
\text { If } U=\frac{\phi^{4}(-q)}{\phi^{4}\left(-q^{3}\right)} \text { and } V=\frac{\psi^{4}(q)}{\psi^{4}\left(q^{3}\right)} \text {, then } U-U V=9-V . \\
\text { If } M=\frac{f^{2}(-q)}{q^{\frac{1}{6}} f^{2}\left(-q^{3}\right)} \text { and } N=\frac{f^{2}\left(-q^{2}\right)}{q^{\frac{1}{3}} f^{2}\left(-q^{6}\right)}, \\
\text { then } M N+\frac{9}{M N}=\left(\frac{N}{M}\right)^{3}+\left(\frac{M}{N}\right)^{3} . \\
\text { If } U=\frac{\phi^{4}(q)}{\phi^{4}\left(q^{3}\right)} \text {, then } \frac{f^{4}(q) f^{4}\left(-q^{2}\right)}{q f^{4}\left(q^{3}\right) f^{4}\left(-q^{6}\right)}=\frac{U(U-9)}{1-U}, \quad U \neq 1 .
\end{gathered}
$$

\section{The Main Results}

Here we state and prove certain interesting interrelations among $q$-product identities and combinatorial partition identities.

Theorem 3.1. Each of the following relations holds true:

$$
\begin{aligned}
& \left(9-\frac{1}{q}\left\{\frac{R(1,1,1,1,1,2)}{R(3,3,1,1,1,2)}\right\}^{4}\right)=\left(1-\frac{1}{q}\left\{\frac{R(1,1,1,1,1,2)}{R(3,3,1,1,1,2)}\right\}^{4}\right) \times \\
& \times \frac{\left(q, q, q, q, q^{2}, q^{2}, q^{2}, q^{2} ; q^{2}\right)_{\infty}\left(-q^{3},-q^{3},-q^{3},-q^{3},-q^{6},-q^{6},-q^{6},-q^{6} ; q^{6}\right)_{\infty}}{\{R(2,1,1,1,2,2) R(2,2,1,1,2,2)\}^{4}\left(q^{3}, q^{3}, q^{3}, q^{3}, q^{6}, q^{6}, q^{6}, q^{6} ; q^{6}\right)_{\infty}}, \\
& \frac{\left(q, q, q^{2}, q^{2}, q^{2}, q^{2} ; q^{2}\right)_{\infty}}{q^{\frac{1}{2}}\left(q^{3}, q^{3}, q^{6}, q^{6}, q^{6}, q^{6} ; q^{6}\right)_{\infty}}+\frac{9 q^{\frac{1}{2}}\left(q^{3}, q^{3}, q^{6}, q^{6}, q^{6}, q^{6} ; q^{6}\right)_{\infty}}{\left(q, q, q^{2}, q^{2}, q^{2}, q^{2} ; q^{2}\right)_{\infty}}= \\
& (18)=\frac{1}{q^{\frac{1}{2}}}\left\{\frac{R(1,1,1,1,1,2) R(12,12,1,1,1,2)\left(q^{3} ; q^{6}\right)_{\infty}\left(q^{6} ; q^{12}\right)_{\infty}\left(q^{12} ; q^{24}\right)_{\infty}}{\left(q^{2} ; q^{2}\right)_{\infty}\left(q^{6} ; q^{12}\right)_{\infty}\left(q^{24} ; q^{24}\right)_{\infty}}+\right. \\
& +q^{\frac{1}{2}}\left\{\frac{R(2,2,1,1,1,2) R(3,3,1,1,1,2)\left(q ; q^{2}\right)_{\infty}\left(q^{2} ; q^{4}\right)_{\infty}}{\left(q^{4} ; q^{4}\right)_{\infty}\left(q^{6} ; q^{6}\right)_{\infty}}\right\}^{6}, \\
& \frac{(-q,-q,-q,-q ;-q)_{\infty}\left(q^{2}, q^{2}, q^{2}, q^{2} ; q^{2}\right)_{\infty}}{q \cdot\left(-q^{3},-q^{3},-q^{3},-q^{3} ;-q^{3}\right)_{\infty}\left(q^{6}, q^{6}, q^{6}, q^{6} ; q^{6}\right)_{\infty}}= \\
& =\frac{\left\{R(2,1,1,1,2,2) \cdot R(1,1,1,1,1,2)\left(q^{3},-q^{6} ; q^{6}\right)_{\infty}\right\}^{4}}{\left\{\left(-q^{3}, q^{6} ; q^{6}\right)_{\infty}\right\}^{4}} \times \\
& \times \frac{\left\{R(1,1,1,1,1,2) R(2,1,1,1,2,2)\left(q^{3},-q^{6} ; q^{6}\right)_{\infty}\right\}^{4}-9\left\{R(2,2,1,1,1,2)\left(-q^{3}, q^{6} ; q^{6}\right)\right\}^{4}}{\left\{R(2,2,1,1,2,2)\left(-q^{3}, q^{6} ; q^{6}\right)_{\infty}\right\}^{4}} \times \\
& \times \frac{\left\{\left(-q^{3}, q^{6} ; q^{6}\right)_{\infty}\right\}^{4}}{\left\{R(2,2,1,1,1,2)\left(-q^{3}, q^{6} ; q^{6}\right)_{\infty}\right\}^{4}-\left\{R(1,1,1,1,1,2) R(2,1,1,1,2,2)\left(q^{3}, q^{6} ; q^{6}\right)\right\}^{4}}
\end{aligned}
$$


Proof. First of all we have to prove our first identity (17). Applying (4)-(5), and further using (11)-(13), we get

$$
\begin{aligned}
U= & \frac{\phi^{4}(-q)}{\phi^{4}\left(-q^{3}\right)}=\frac{\left(q, q, q, q, q^{2}, q^{2}, q^{2}, q^{2} ; q^{2}\right)_{\infty}}{\{R(2,1,1,1,2,2) R(2,2,1,1,2,2)\}^{4}} \times \\
& \times \frac{\left(-q^{3},-q^{3},-q^{3},-q^{3},-q^{6},-q^{6},-q^{6},-q^{6} ;-q^{6}\right)_{\infty}}{\left(q^{3}, q^{3}, q^{3}, q^{3}, q^{6}, q^{6}, q^{6}, q^{6} ; q^{6}\right)_{\infty}}
\end{aligned}
$$

and

$$
\begin{gathered}
V=\frac{\psi^{4}(q)}{q \psi^{4}\left(q^{3}\right)}=\frac{1}{q}\left\{\frac{R(1,1,1,1,1,2)}{R(3,3,1,1,1,2)}\right\}^{4}, \\
U V=\frac{\left(q, q, q, q, q^{2}, q^{2}, q^{2}, q^{2} ; q^{2}\right)_{\infty}\{R(1,1,1,1,1,2)\}^{4}}{\{R(2,1,1,1,2,2) R(2,2,1,1,2,2)\}^{4}} \times \\
\times \frac{\left(-q^{3},-q^{3},-q^{3},-q^{3},-q^{6},-q^{6},-q^{6},-q^{6} ;-q^{6}\right)_{\infty}}{q \cdot\left(q^{3}, q^{3}, q^{3}, q^{3}, q^{6}, q^{6}, q^{6}, q^{6} ; q^{6}\right)_{\infty}\{R(3,3,1,1,1,2)\}^{4}}, \\
U-U V=\left(1-\frac{1}{q}\left\{\frac{R(1,1,1,1,1,2)}{R(3,3,1,1,1,2)}\right\}^{4}\right) \times \\
\times \frac{\left(q, q, q, q, q^{2}, q^{2}, q^{2}, q^{2} ; q^{2}\right)_{\infty}}{\{R(2,1,1,1,2,2) R(2,2,1,1,2,2)\}^{4}} \times \\
\times \frac{\left(-q^{3},-q^{3},-q^{3},-q^{3},-q^{6},-q^{6},-q^{6},-q^{6} ;-q^{6}\right)_{\infty}}{\left(q^{3}, q^{3}, q^{3}, q^{3}, q^{6}, q^{6}, q^{6}, q^{6} ; q^{6}\right)_{\infty}}
\end{gathered}
$$

using (20)-(23) into (14), we obtain required identity (17).

Further, we have to attempt to estabilish our second identity (18). Applying (6), we obtain:

$$
M=\frac{f^{2}(-q)}{q^{\frac{1}{6}} f^{2}\left(-q^{3}\right)}=\frac{\left\{(q ; q)_{\infty}\right\}^{2}}{q^{\frac{1}{6}}\left\{\left(q^{3} ; q^{3}\right)_{\infty}\right\}^{2}}
$$

and

$$
N=\frac{f^{2}\left(-q^{2}\right)}{q^{\frac{1}{3}} f^{2}\left(-q^{6}\right)}=\frac{\left\{\left(q^{2} ; q^{2}\right)_{\infty}\right\}^{2}}{q^{\frac{1}{3}}\left\{\left(q^{6} ; q^{6}\right)_{\infty}\right\}^{2}}
$$

with (24)-(25), and using (11)-(13), we have following identities

$$
M N=\frac{\left(q, q, q^{2}, q^{2}, q^{2}, q^{2} ; q^{2}\right)_{\infty}}{q^{\frac{1}{2}}\left(q^{3}, q^{3}, q^{6}, q^{6}, q^{6}, q^{6} ; q^{6}\right)_{\infty}}
$$

and

$$
\frac{9}{M N}=\frac{9 q^{\frac{1}{2}}\left(q^{3}, q^{3}, q^{6}, q^{6}, q^{6}, q^{6} ; q^{6}\right)_{\infty}}{\left(q, q, q^{2}, q^{2}, q^{2}, q^{2} ; q^{2}\right)_{\infty}}
$$


Combining (26) and (27) we get

$$
M N+\frac{9}{M N}=\frac{\left(q, q, q^{2}, q^{2}, q^{2}, q^{2} ; q^{2}\right)_{\infty}}{q^{\frac{1}{2}}\left(q^{3}, q^{3}, q^{6}, q^{6}, q^{6}, q^{6} ; q^{6}\right)_{\infty}}+\frac{9 q^{\frac{1}{2}}\left(q^{3}, q^{3}, q^{6}, q^{6}, q^{6}, q^{6} ; q^{6}\right)_{\infty}}{q^{\frac{1}{2}}\left(q, q, q^{2}, q^{2}, q^{2}, q^{2} ; q^{2}\right)_{\infty}}
$$

Also, we have

$$
\begin{aligned}
\frac{M}{N} & =q^{\frac{1}{6}}\left\{\frac{\left(q ; q^{2}\right)_{\infty}\left(q^{6} ; q^{6}\right)_{\infty}\left(q^{2} ; q^{2}\right)_{\infty}}{\left(q^{2} ; q^{2}\right)_{\infty}\left(q^{3} ; q^{6}\right)_{\infty}\left(q^{6} ; q^{6}\right)_{\infty}}\right\}^{2}= \\
& =q^{\frac{1}{6}}\left\{\frac{R(2,2,1,1,1,2) R(3,3,1,1,1,2)\left(q ; q^{2}\right)_{\infty}\left(q^{2} ; q^{4}\right)_{\infty}}{\left(q^{4} ; q^{4}\right)_{\infty}\left(q^{6} ; q^{6}\right)_{\infty}}\right\}^{2}
\end{aligned}
$$

Similarly,

$$
\frac{N}{M}=\frac{1}{q^{\frac{1}{6}}}\left\{\frac{R(1,1,1,1,1,2) R(12,12,1,1,1,2)\left(q^{3} ; q^{6}\right)_{\infty}\left(q^{6} ; q^{12}\right)_{\infty}\left(q^{12} ; q^{24}\right)_{\infty}}{\left(q^{2} ; q^{2}\right)_{\infty}\left(q^{6} ; q^{12}\right)_{\infty}\left(q^{24} ; q^{24}\right)_{\infty}}\right.
$$

Combining (29) and (30), we get

$$
\begin{aligned}
& \left(\frac{N}{M}\right)^{3}+\left(\frac{M}{N}\right)^{3}= \\
= & \frac{1}{q^{\frac{1}{2}}}\left\{\frac{R(1,1,1,1,1,2) R(12,12,1,1,1,2)\left(q^{3} ; q^{6}\right)_{\infty}\left(q^{6} ; q^{12}\right)_{\infty}\left(q^{12} ; q^{24}\right)_{\infty}}{\left(q^{2} ; q^{2}\right)_{\infty}\left(q^{6} ; q^{12}\right)_{\infty}\left(q^{24} ; q^{24}\right)_{\infty}}\right\}^{6}+ \\
& +q^{\frac{1}{2}}\left\{\frac{R(2,2,1,1,1,2) R(3,3,1,1,1,2)\left(q ; q^{2}\right)_{\infty}\left(q^{2} ; q^{4}\right)_{\infty}}{\left(q^{4} ; q^{4}\right)_{\infty}\left(q^{6} ; q^{6}\right)_{\infty}}\right\}^{6},
\end{aligned}
$$

equating (28) and (31), as precondition given in (15), we obtain the required identity (18).

Finally, we have to prove our third identity (19). In order to establish this identity, using(4) and (6), and further applying (11)-(13), we get

$$
U=\left\{\frac{R(2,1,1,1,2,2) R(1,1,1,1,1,2)\left(q^{3},-q^{6} ; q^{6}\right)_{\infty}}{R(2,2,1,1,2,2)\left(-q^{3}, q^{6} ; q^{6}\right)_{\infty}}\right\}^{4},
$$

from which it follows

$$
\begin{aligned}
& \Rightarrow \frac{U^{2}-9 U}{1-U}= \\
& \frac{\left\{R(2,1,1,1,2,2) R(1,1,1,1,1,2)\left(q^{3},-q^{6} ; q^{6}\right)_{\infty}\right\}^{4}}{\left\{\left(-q^{3}, q^{6} ; q^{6}\right)_{\infty}\right\}^{4}} \times \\
& \times \frac{\left\{R(1,1,1,1,1,2) R(2,1,1,1,2,2)\left(q^{3},-q^{6} ; q^{6}\right)_{\infty}\right\}^{4}-9\left\{R(2,2,1,1,1,2)\left(-q^{3}, q^{6} ; q^{6}\right)\right\}^{4}}{\left\{R(2,2,1,1,2,2)\left(-q^{3}, q^{6} ; q^{6}\right)_{\infty}\right\}^{4}} \times \\
& \times \frac{\left\{\left(-q^{3}, q^{6} ; q^{6}\right)_{\infty}\right\}^{4}}{\left\{R(2,2,1,1,1,2)\left(-q^{3}, q^{6} ; q^{6}\right)_{\infty}\right\}^{4}-\left\{R(1,1,1,1,1,2) R(2,1,1,1,2,2)\left(q^{3},-q^{6} ; q^{6}\right)\right\}^{4}} .
\end{aligned}
$$

Similarly, holds

$$
\frac{f^{4}(q) f^{4}\left(-q^{2}\right)}{q f^{4}\left(q^{3}\right) f^{4}\left(-q^{6}\right)}=\frac{(-q,-q,-q,-q ;-q)_{\infty}\left(q^{2}, q^{2}, q^{2}, q^{2} ; q^{2}\right)_{\infty}}{q \cdot\left(-q^{3},-q^{3},-q^{3},-q^{3} ;-q^{3}\right)_{\infty}\left(q^{6}, q^{6}, q^{6}, q^{6} ; q^{6}\right)_{\infty}},
$$

equating (32) and (33), using in (16), we obtain (19). Hence, we complete proof of our theorem. 


\section{Concluding Remarks and Observations}

The present investigation was motivated by several recent developments dealing essentially with $q$-product identities and combinatorial partition identities. Here, in this article, we have established three presumably new inter-relationships that exist among $q$-product identities and combinatorial partition identities. We have also considered several closely-related identities such as (for example) q-product identities and Jacobi's triple-product identities. We have chosen to indicate rather briefly a number of recent developments on the subject-matter of this article.

The list of citations, which we have included in this article, is believed to be potentially useful for indicating some of the directions for further researches and related developments on the subject-matter which we have dealt with here, and in particular, the recent works by Chaudhary et al. (see $[11,5,8,9,7,6,10])$.

\section{Conflicts of Interest}

All three authors declare that they have no conflict of interest.

\section{ACKNOWLEDGEMENT}

The research work of the second named author (Sangeeta Chaudhary) has been sponsored by the UGC Dr. D. S. Kothari Post Doctoral Fellowship Scheme by its letter ref No. F.4-2/2006 (BSR)/MA/18-19/0022.

\section{REFERENCES}

[1] C. Adiga, T. Kim, M. S. Mahadeva Naika and H. S. Madhusudhan, On Ramanujan's cubic continued fraction and explicit evaluations of theta functions, Indian Journal of Pure and Applied Mathematics, 35(9) (2004), 1047-1062.

[2] G. E. Andrews, K. Bringman and K. Mahlburg, Double series representations for Schur's partition function and related identities, Journal of Combinatorial Theory Series A, 132 (2015), 102-119.

[3] B. C. Berndt, Ramanujan's Notebooks, Part III, Springer-Verlag, Berlin, Heidelberg and New York, 1991.

[4] N. D. Baruah and J. Bora, Modular relations for the nonic analogues of the Rogers Ramanujan functions with applications to partitions, Journal of Number Theory, 128 (2008), 175-206.

[5] M. P. Chaudhary, Some relationships between q-product identities, combinatorial partition identities and continued-fractions identities III, Pacific Journal of Applied Mathematics, 7(2) (2015), 87-95.

[6] M. P. Chaudhary and G. A. Salilew, Two relations between q-product identities and combinatorial partition identities, Pacific Journal of Applied Mathematics, 8(4) (2017), 263-274. 
[7] M. P. Chaudhary, G. A. Salilew and J. Choi, Five relationships between continued fraction identities, q-product identities and combinatorial partition identities, Far East Journal of Mathematical Sciences, 102(4) (2017), 855-863.

[8] M. P. Chaudhary and J. Choi, Note on modular relations for Roger-Ramanujan type identities and representations for Jacobi identities, East Asian Mathematical Journal 31(5) (2015), 659-665.

[9] M. P. Chaudhary and J. Choi, Certain identities associated with character formulas, continued fractions and combinatorial partition, identities, East Asian Mathematical Journal, 32(5) (2016) 609-619.

[10] M. P. Chaudhary and J. Choi, Representations of Ramanujan continued fraction in terms of combinatorial partition identities, Honam Mathematical Journal, 38(2) (2016), 367-373.

[11] M. P. Chaudhary and J. L. Cimadevilla Villacorta, Representations of certain theta function identities in terms of combinatorial partition identities, Far East Journal of Mathematical Sciences, 102(8) (2017), 1605-1611.

[12] M. S. Mahadeva Nakia, K. S. Bairy and N. P. Suman, Certain modular relations for remarkable product of theta- functions, Proceedings of the Jangeon Mathematical Society, 17(3) (2014), 317-331.

[13] H. M. Srivastava and M. P. Chaudhary, Some relationships between q-product identities, combinatorial partition identities and continued-fractions identities, Advanced Studies in Contemporary Mathematics, 25(3) (2015), 265-272.

[14] H. M. Srivastava, M. P. Chaudhary and S. Chaudhary, A family of theta function identities related to Jacobi's triple-product identity, Russian Journal of Mathematical Physics, 27(1) (2020), 139-144.

[15] H. M. Srivastava and J. Choi, Zeta and q-Zeta Functions and Associated Series and Integrals, Elsevier Science Publishers, Amsterdam, London and New York, 2012.

M.P. Chaudhary

Department of Mathematics

Netaji Subhas University of Technology

New Delhi 110078

INDIA

E-mail address: dr.m.p.chaudhary@gmail.com

SANGeeta Chaudhary

School of Computater and Systems Sciences

JaWAHARLAL NeHRU UNIVERSITY

NeW Delhi 110067

INDIA

Sonajharia Minz

School of Computater and Systems Sciences

JaWAharlal Nehru University

New Delhi 110067

INDIA 